Journal of Science and Technology Research

Journal homepage: www.nipesjournals.org.ng

NIPES

\title{
Corrosion Analysis of API 5L Gr. Three Phase Crude
}

Artford Usuoyibo ${ }^{1}$, Obogai L.E ${ }^{2}$

${ }^{1}$ Department of Mechanical Engineering, University of Benin, Nigeria, P.M.B 1154, Benin City

${ }^{2}$ Department of Mechanical Engineering, Federal University Utuoke, P.M.B 126, Yenagoa

\section{Article Info}

Received 18 July 2020

Revised 29 July 2020

Accepted 30 July 2020

Available online 31 August 2020

Keywords:

Pipeline, Magnetic Flux Leakage, Pigging, Corrosion, Pipeline Integrity Management

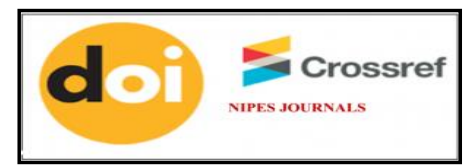

https://doi.org/10.37933/nipes/2.3.2020.21

https://nipesjournals.org.ng (C) 2020 NIPES Pub. All rights reserved

\begin{abstract}
The safest and most economical way of transporting crude oil is the use of steel pipes; however, pipelines have continually failed from corrosion as a result of the poor analysis of data recorded from the intelligent pigging of the pipeline. In this work, two ultrasonic Pipeline Internal Gauge PIGs and one magnetic flux leakage PIG were used to obtain data from three phase crude (carrying a mixture of crude oil, water and gas) from Mimbo to TMMP. The pipelines are located in the Niger Delta region of Nigeria. The ultrasonic PIG uses the pulse-echo principle to determine the wall thickness of steel pipe walls while the magnetic flux leakage PIG measures the changes in the magnetic field close to the pipe wall. A cleaning PIG" was first run through the pipelines to ensure that the pipelines were free of debris that may alter the signals and data collected by the intelligent PIGs (smart PIGs). The collected data from the smart PIGs runs were analysed using Dacon Technology Software and the results obtained was compared with the line pipe design data obtained from international standards and codes to determine the status of the service condition of the operating pipeline systems. The Estimated Repair Factor (ERF) results were the basis for determining the service condition of the pipeline. The result showed that MFL intelligent pigging reduced the metal loss in the pipeline transporting three (3) phase crude from Mimbo to TMMP by $17.11 \%$ between 2005 and 2015 pigging periods. The metal loss peak data showed that the pipeline transporting three phase crude from Mimbo to TMMP passed through three separate environments which have different corrosion rate on the pipeline. From the data collected and analyzed, it was observed that the internal conditions of the pipeline have more effect on the ERF. It was also observed from the data obtained that the points on the pipeline from Mimbo to TMMP transporting liquid crude with $E R F \geq 0.2$ have anomalies at the internal wall of the pipeline.
\end{abstract}

\section{Introduction}

Pipes are tubular section or hollow cylinder, usually but not necessarily of circular cross-section, used to convey or transport mainly fluids (liquids and gases) from one point to the other [1]. Pipes are made with metal or plastics and find applications both domestically and industrially and their importance cannot be over emphasized. In the oil and gas industry, pipes represent the nerve system of the oil and gas industry as they are used in transporting petroleum from the oil wells through to the points of sales. Steel pipes are used in the transportation of petroleum and their integrity cannot be compromised if the oil and gas industry is to remain functional. Pipelines are 40 times safer than rail tanks, and 100 times safer than road tanks in transporting crude oil [2]. 
Corrosion, being the fundamental cause of pipeline failure in the oil and gas industry, minimizing or possibly eliminating it will inevitably result in increased production as well as eliminating the threats posed from it. A proper and adequate monitoring of pipeline corrosion is imperative in avoiding disasters that may be catastrophic. For effective monitoring of pipeline reliability and remaining service life prediction, corrosion risk assessment and management are necessary. One way of assessing corrosion risk of pipeline integrity is the use of Pipeline Integrity Management (PIM) [3]. With the enormous wealth generated from the oil and gas industry, proactive and preventive steps must be taken to ensure its continuous operations. The oil and gas industry are arguably one of the biggest industries worldwide and it is highly dependent on pipes and pipeline networks which is the cheapest and most effective means of transporting its products i.e. crude oil and its products. These pipes and pipeline networks are an integral part of the oil and gas industry; however, they are threatened by corrosion in all its forms, leading to failure of these pipes during service. The safety of these pipes during operation must be guaranteed. Pipeline Integrity Management (PIM) involves the understanding and operation of pipelines in a safe and reliable manner [4]. Pipe corrosion is one of the significant threats to safety in the operations of the oil and gas industry. It is important to have a system that checks and prevents these threats from becoming reality. The increased need for energy and the attendant pressure on the oil and gas pipelines entails that the integrity of these pipelines be maintained to minimize non-productive time, increase the efficiency of the oil and gas transportation and protect the environment. Pipeline Integrity Management involves the identification of process for understanding pipeline failure mode, assessment path for the failure mode, integrity analysis procedure for failure and consequences, setting criteria for repair action and information analysis, continuous process of assessment to keep maintaining integrity, mitigation and preventive actions to forestall downtime, effectiveness measure and review techniques [5].

The safety and integrity of these pipes must be ensured and constantly monitored and maintained to allow the pipes live their design life. Pipeline Integrity Management (PIM) is the cradle-to-grave approach of understanding and operating oil and gas pipe and pipeline networks in a safe and reliable manner [5]. It is a systematic process in the monitoring and inspection of oil and gas pipelines in the prevention of failures as well as predicting possible points of failure in pipelines through the collection and analysis of data from oil and gas pipelines. Pigging is one of the methods used to monitor the integrity of oil and gas pipelines. PIG (Pipeline Inspection Gauge) as it is commonly called is a machine which is used to collect data from oil and gas pipelines in service. Data collected by PIG machines require proper and adequate analysis for effective pipeline maintenance.

Magnetic Flux Leakage (MFL) induces a magnetic field to the pipe. As it travels, it locates and records magnetic flux anomalies in the pipe. The recorded magnetic flux data is converted to information that provides an indication of metal loss in the pipe. There are two types of these tools, High Resolution MFL and Standard Resolution MFL. The main difference between the two is in the number of sensors and the amount of resolution [6]. Most MFL tools can determine the location and at 6 O'clock position of the metal loss anomaly and detect if a corrosion anomaly is internal or external to the pipe wall. It also provides data of each corrosion anomaly including its length and maximum pit depth, which allows for calculation to determine the pipe's remaining strength. MFL pigs are generally capable of detecting corrosion greater than $20 \%$ of the pipe wall thickness in depth; however, axially-oriented flaws such as stress corrosion cracking, selective seam corrosion and axial gouges are difficult to detect with MFL pigs [7].

Ultrasonic also called a "UT tool", this tool provides similar physical pipe data as the MFL tool, but it uses ultrasonic technology. The UT tool transmits an ultrasonic pulse into the pipe wall and directly measures its thickness. Since this technology requires a clean pipe wall, it is generally not used for certain pipelines such as crude lines with a paraffin build-up. There are also wall-thickness limitations with the UT tool. It works well with heavy-wall pipe, but not as well with thin-wall pipe, and it is not as widely used as the MFL tool [8]. 


\section{Methodology}

The materials and methods used in the analysis of the pigging data collected are discussed here. Three smart pig ILI data were analysed, one magnetic flux leakage (MFL) and the other two are ultrasonic (UT). The data are obtained from an existing oil and gas operating pipelines in the Niger Delta region of Nigeria. The MFL pig was used to obtain data from an API 5L Gr. X56 steel pipeline which is operating three phase crudes in OML 23, from MIMBO to TMMP. The data collected by the MFL were the "wall thickness" at 50 different locations along the pipeline and thrice over a period of 15 years. The first UT PIG was used to obtain data from a 4inch straight seam welded API 5L Gr. B steel pipe; schedule 40, 60, 80 thickness $6.02 \mathrm{~mm}, 7.14 \mathrm{~mm}, 8.56 \mathrm{~mm}$, and is operating crude oil in Mimbo to TMMP, while the second UT PIG was used to obtain data from a 4inch API 5L Gr. B steel pipeline which is operating gas in Mimbo to TMMP. The parameter obtained by the three smart PIGs were the "up weld distance", "identified anomaly", "anomaly class", "nominal thickness", "anomaly length", "anomaly width" and "anomaly surface location". The calculated parameters were the "depth" which is the percentage of metal loss, and the "estimated repair factor" which indicates the level of anomaly. The collected data were analyzed using Dacon Technology Software and Microsoft Excel program. The data obtained were analysed to determine the parameter which is most important in determining the continued service condition of oil and gas API 5L steel pipes.

\section{Results and Discussion}

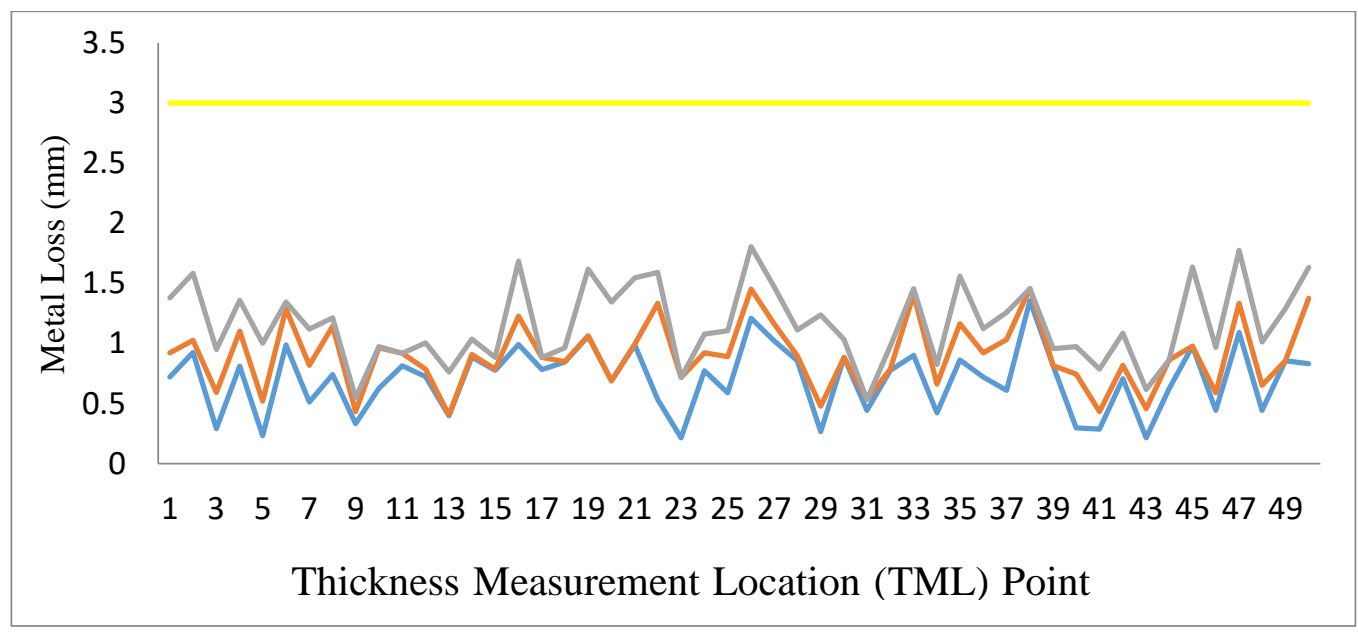

Key:

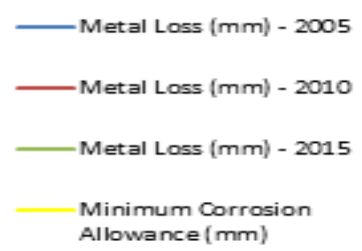

Figure 1: Plot of metal loss and the minimum corrosion allowance for the three successive in-lineinspections carried out on the three-phase crude pipeline 
Artford Usuoyibo, Obogai L.E / NIPES Journal of Science and Technology Research 2(3) 2020 pp. 199 - 207

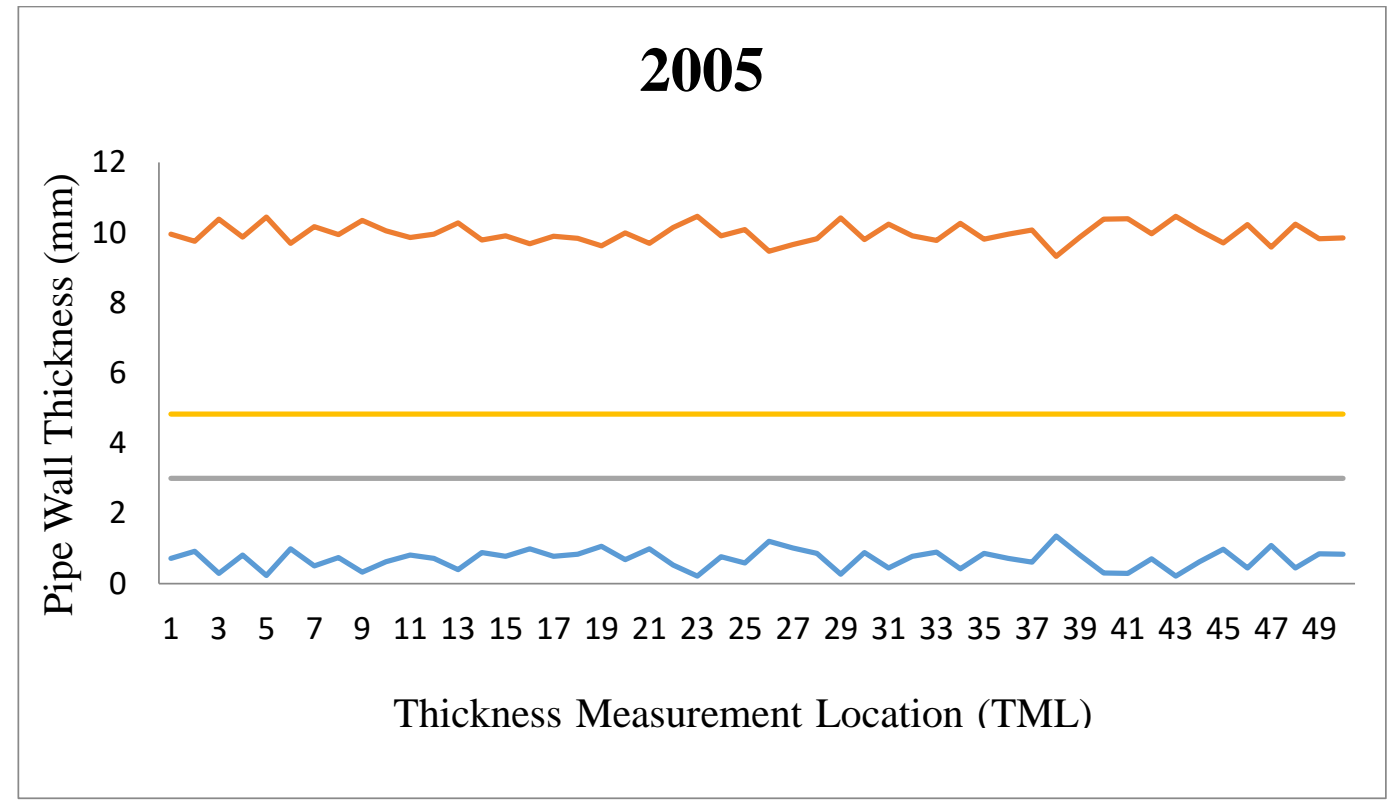

Key:

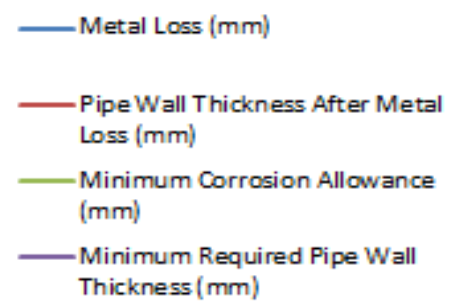

Figure 2: Plot of metal loss and remaining wall thickness against minimum specified standards after the first intelligent pigging in 2005

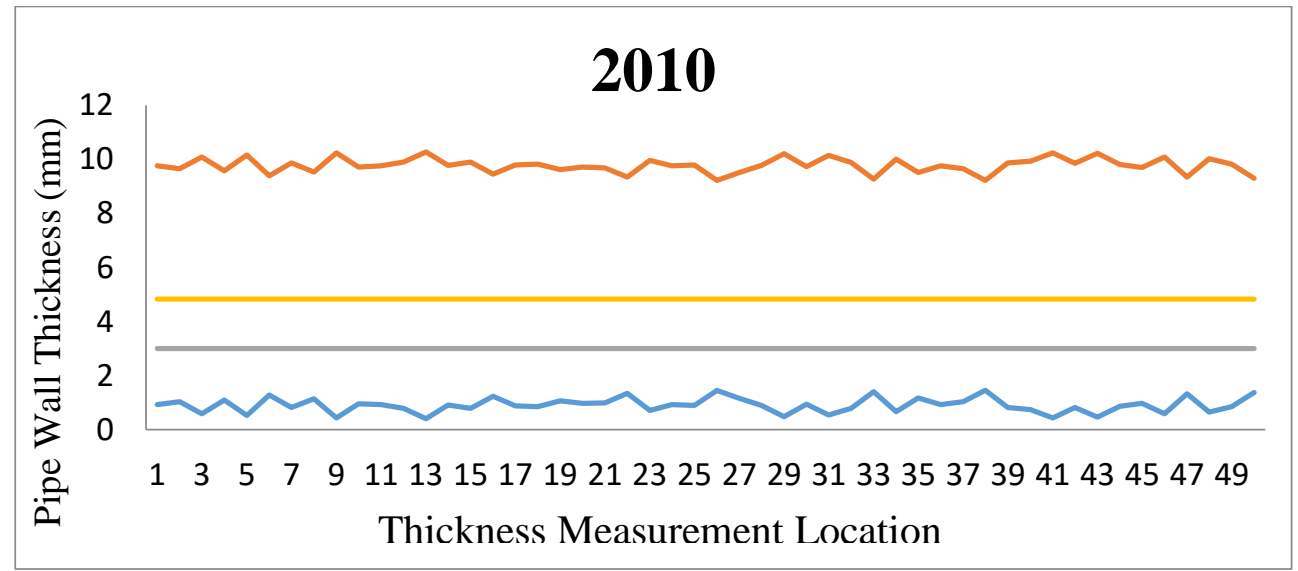

Key:

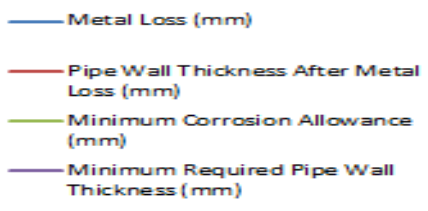

Figure 3: Plot of metal loss and remaining wall thickness against minimum specified standards after the first intelligent pigging in 2010 


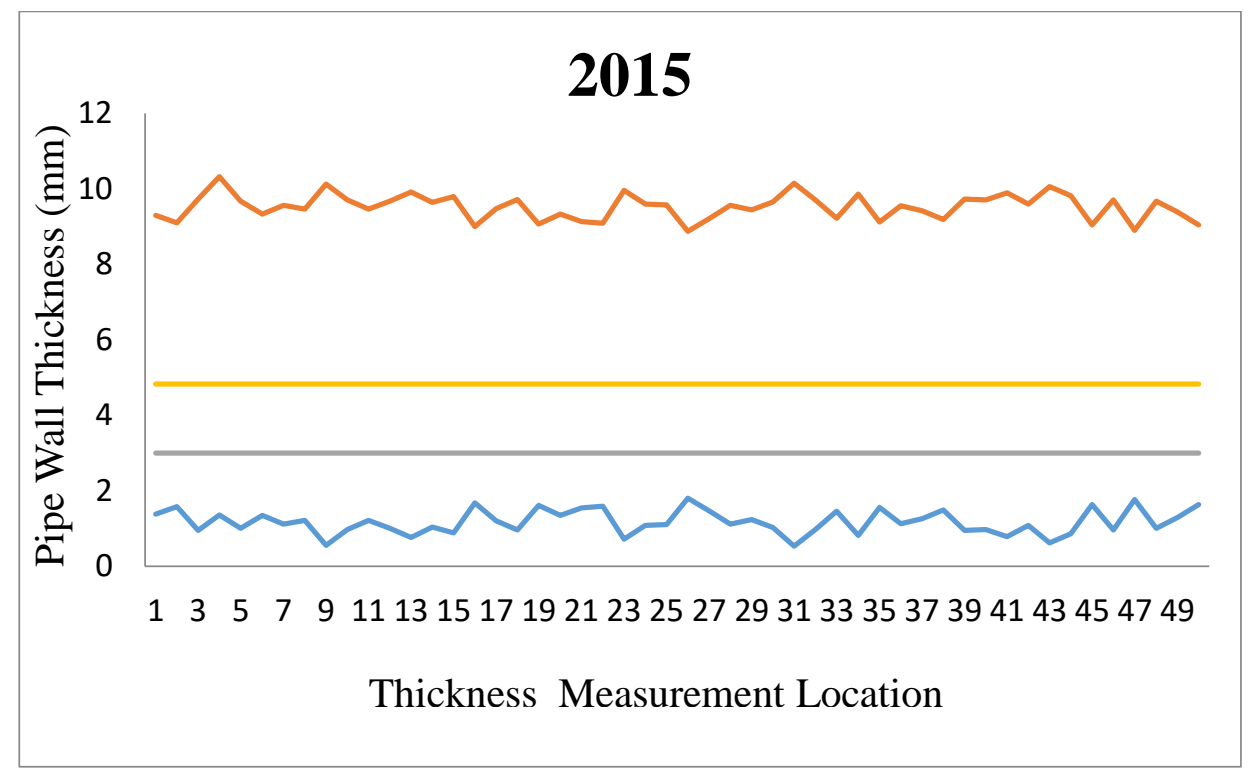

\section{Key:}

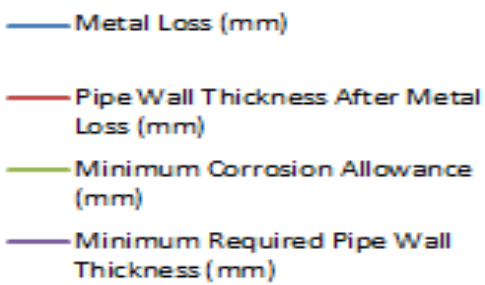

Figure 4: Plot of metal loss and remaining wall thickness against minimum specified standards after the first intelligent pigging in 2015.

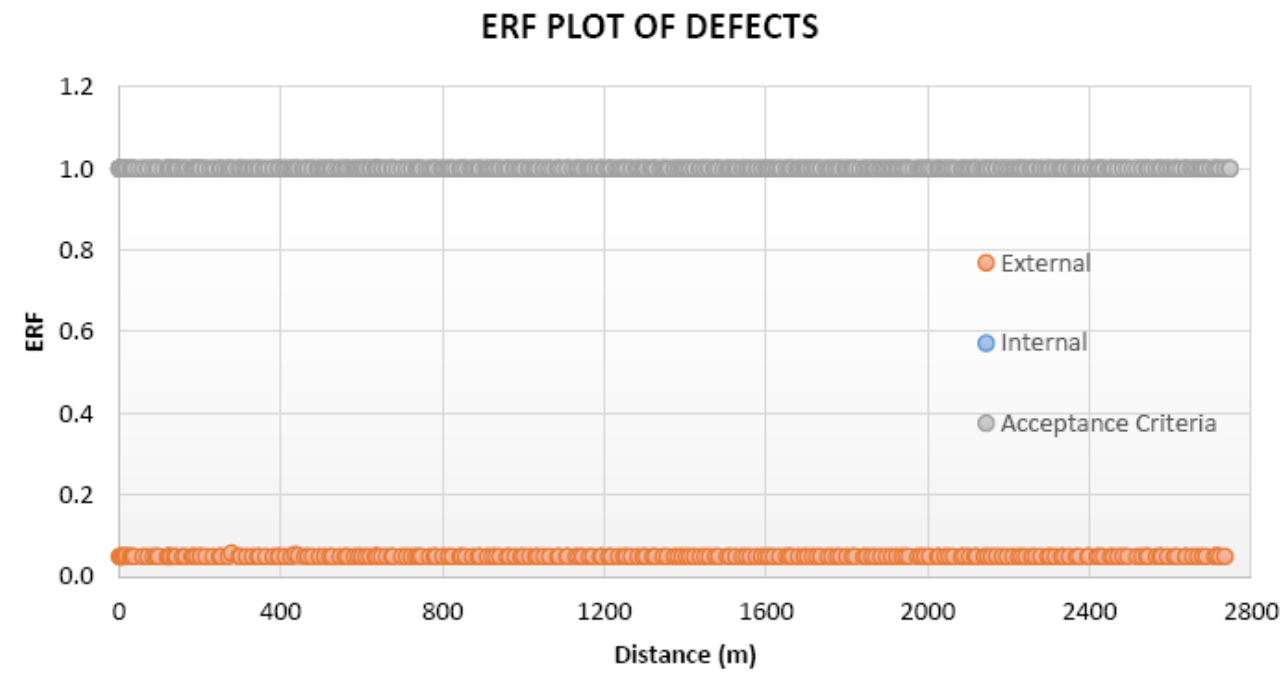

Figure 5: Plot of estimated repair factor (ERF) of defects along pipeline 
THICKNESS

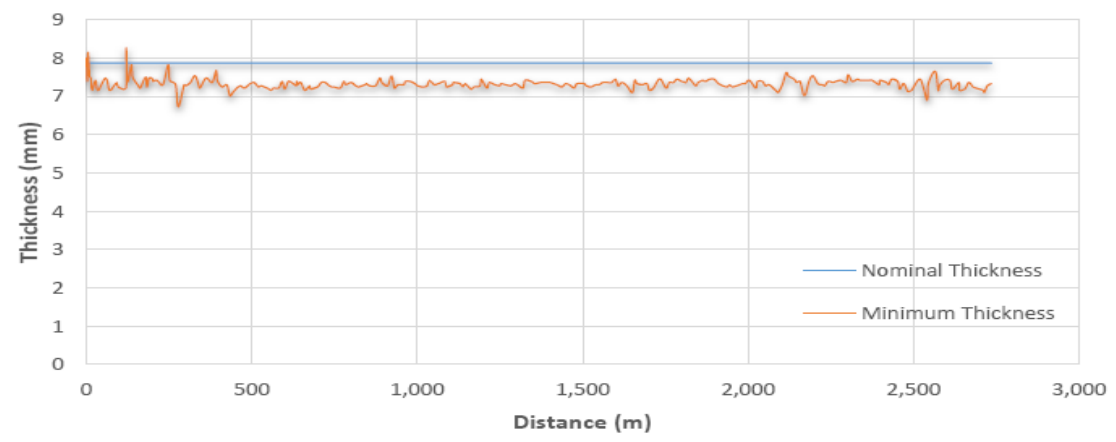

Figure 6: Plot of average thickness compared with the nominal thickness of the pipe

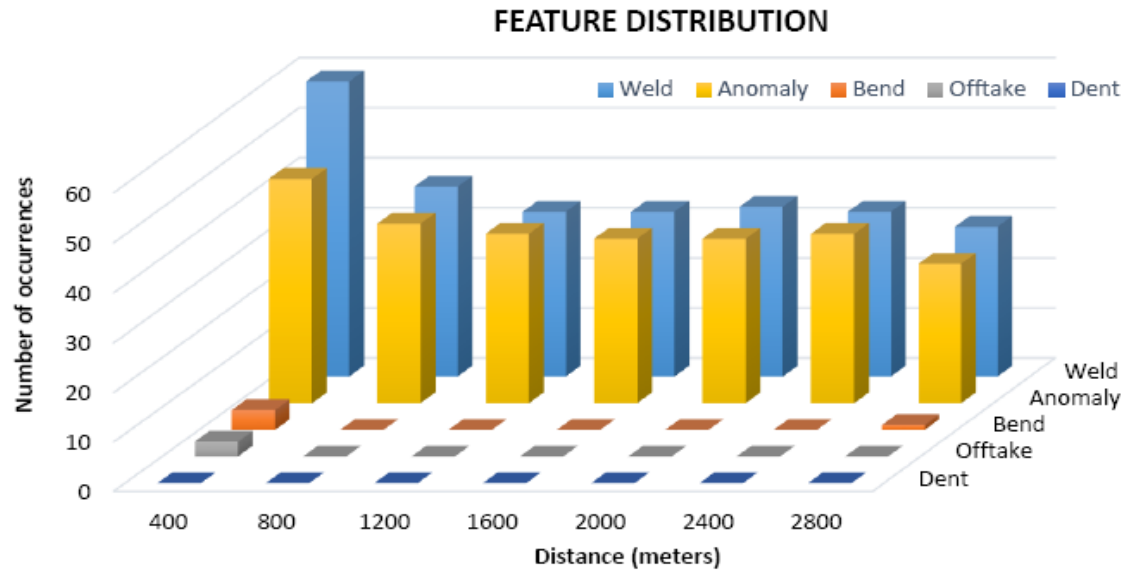

Figure 7: Plot of feature distribution along pipeline

\section{METAL LOSS PEAK DEPTH DISTRIBUTION PLOTS}

口 $60 \% \leq d<70 \% \quad 40 \% \leq d<50 \% \quad \square 0 \% \leq d<40 \% \quad 20 \% \leq d<30 \% \quad \square \quad 10 \% \leq d<20 \% \quad-d<10 \%$

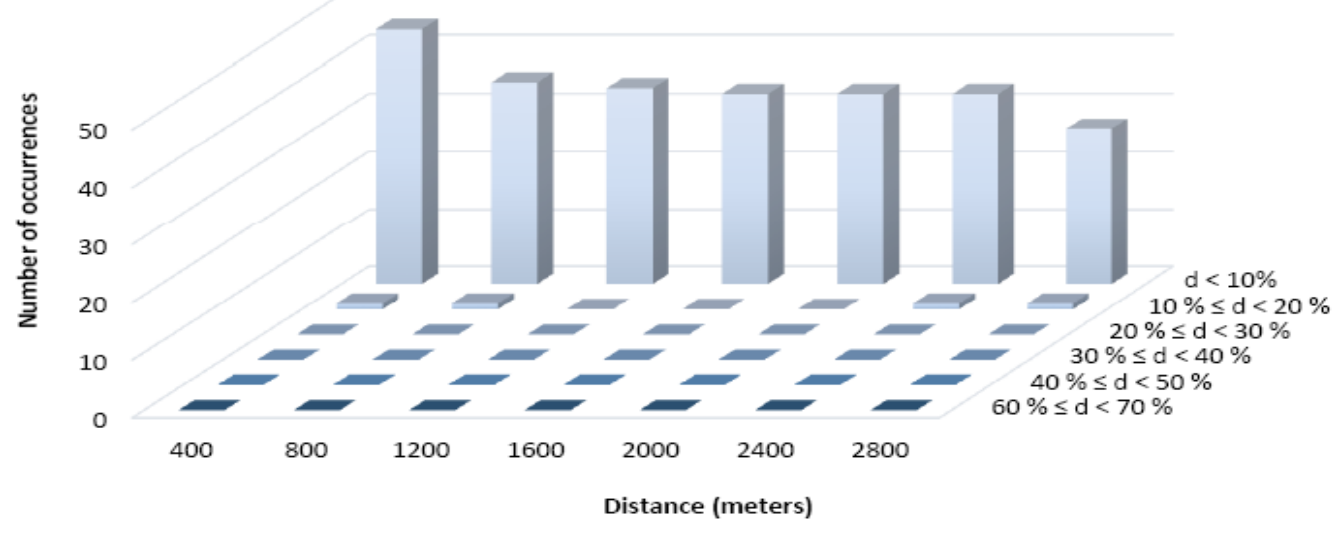

Figure 8: Metal loss peak depth distribution plot 


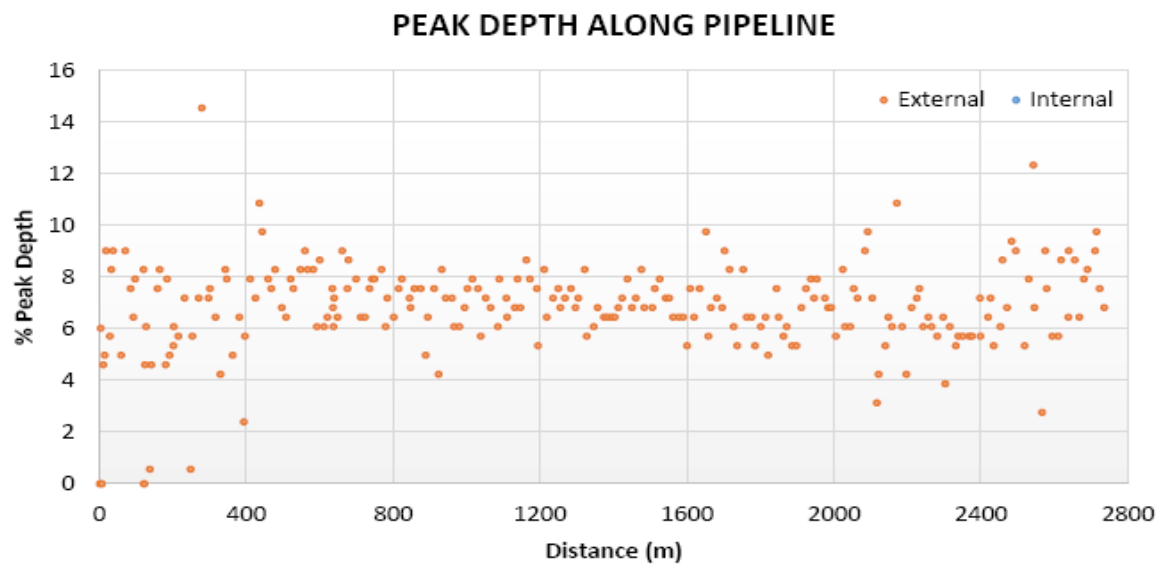

Figure 9: Peak depth along pipeline

\section{ANOMALY IDENTIFICATION}

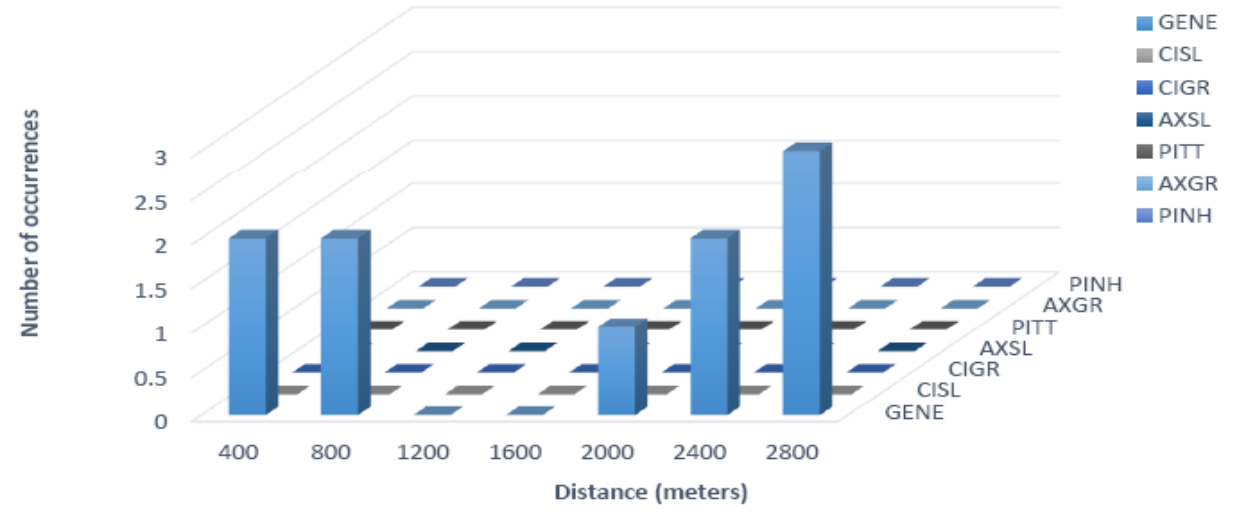

Figure 10: Plot of anomalies identified along the pipeline

From Figure 1, the metal loss recorded for the three pigging periods along the pipeline showed an irregular curve pattern indicating a stochastic corrosion pattern along the pipeline. The minimum corrosion allowance is shown in the plot and is seen above the metal loss curves, indicating that the corrosion allowance is not broken and hence the pipeline is safe for continued service operation [3]. Figure 1 shows the average amount of metal loss that occurred at the 50 selected points in the pipeline between the first and second intelligent pigging i.e. 2005 and 2010 , was $0.235 \mathrm{~mm}$, while the average amount of corrosion that occurred between the second and third intelligent pigging i.e. 2010 and 2015 was $0.304 \mathrm{~mm}$. The maximum metal loss from the thickness measurement location (TML) intelligent pigging data obtained was between the period of 2010 and 2015 with a value of $0.761 \mathrm{~mm}$ at "TML Point 29 " and the minimum metal loss from the thickness measurement location (TML) data obtained was between the period of 2010 and 2015 with a value of $0.040 \mathrm{~mm}$ at "TML Point 38\%. There is an average of $17.11 \%$ reduction in the amount of metal loss recorded along the pipeline between 2005 and 2015 pigging period. The result of the reduction of the metal loss may be as a result of the "Cleaning Pig" which is run through the pipeline before it is intelligently pigged using the required and desired PIG. Cleaning PIG is therefore an important criterion that is useful in the reduction of metal loss. The thickness measurement location (TML) intelligent pigging is useful for the monitoring the amount of corrosion via metal loss in a pipeline [5]. Figure 2 shows the amount of metal loss as against the minimum corrosion allowance threshold for the intelligent pigging carried out in the year 2005. It is clearly seen that the metal loss from all 50 thickness measurement locations (TML), the highest value of metal loss is $1.356 \mathrm{~mm}$ at "Point 38 ", corresponding to a minimum pipe wall thickness of $9.324 \mathrm{~mm}$; however, it did not intersect with the 
minimum corrosion allowance threshold of $3 \mathrm{~mm}$ as specified by the ASME standards. This indicates that the pipeline is safe for operation. It is also seen from Figure 2 that the pipe wall thickness after the metal loss was above the minimum required pipe wall thickness of $4.83 \mathrm{~mm}$. From the data collected, the design data and the calculated result, the pipeline is safe for operation with respect to the pipe wall thickness requirement. Figure 2 shows the amount of metal loss, the remaining pipe wall thickness, the corrosion allowance and the minimum required pipe wall thickness after the second intelligent pigging carried out on the pipeline.

As seen Figure 3, the metal loss along the pipeline at all 50 thickness measurement locations (TML) did not intersect the threshold for the minimum corrosion allowance of $3 \mathrm{~mm}$. The highest metal loss was $1.457 \mathrm{~mm}$ at "TML Point 38 " leaving the minimum wall thickness along the pipeline to be $9.223 \mathrm{~mm}$. The pipe wall thickness after metal loss from second intelligent pigging carried out in 2010 did not intersect with the minimum required pipe wall thickness of $4.83 \mathrm{~mm}$. The intelligent pigging data collected when compared with the design data and calculated result indicates the pipeline is deem safe for continued operation with respect to the pipe wall thickness requirement. Figure 4 shows the metal loss, the pipe wall thickness after metal loss, the minimum corrosion allowance and the minimum required pipe wall thickness after the third successive intelligent pigging carried out on the pipeline in 2015 . The maximum metal loss was $1.805 \mathrm{~mm}$ at "TML Point 26 ", leaving the minimum wall thickness along all 50 thickness measurement locations (TML) along the pipeline to be $8.875 \mathrm{~mm}$. The metal loss at all 50 thickness measurement locations (TML) did not intersect the minimum corrosion allowance of $3 \mathrm{~mm}$. The pipe wall thickness after the metal loss also did not intersect the minimum required pipe wall thickness of $4.83 \mathrm{~mm}$. The result indicates that the pipeline is deemed safe for operation with respect to the pipe wall thickness requirement [6].

The estimated repair factor (ERF) of the defects along the pipeline is plotted along the acceptable ERF criteria in Figure 5. The acceptable ERF for the pipeline at any location is $\leq 1$ as required by the ASME B31.4 standard. Any ERF value at any location along the pipeline greater than this value, such location should further be analyzed using a less conservative assessment procedure (e.g. RSTRENG, Finite Element Analysis etc.) or, otherwise, the defect should be repaired using an approved procedure (e.g. replacement of the affected section or encirclement by a pressure retaining clamp); however, from Figure 5, no ERF value along the pipeline exceeded the acceptable value. The highest ERF value recorded was 0.06 .

It is seen in Figure 3.6 that the recorded minimum wall thickness is lower than the nominal thickness as specified by the manufacturer; however, it is below the minimum wall thickness of $4.6 \mathrm{~mm}$, implying that the pipeline is safe for service operation with respect to the pipe wall thickness. The plot of the minimum thickness in Figure 6 is relatively uniform, implying a uniform corrosion on the external walls of the pipeline

Figure 7 show the plot of the feature distribution along the pipeline as recorded by the smart pig. From the pigging data downloaded, this pipeline has 260 welds, 5 bends, 3 off takes, 243 anomalies. There was no dent recorded along the pipeline. The severity of the anomaly with respect to the percentage metal loss along the pipeline is shown Figure 8. From Figure 8, the highest number of anomaly occurrence occurs at a distance of $0-800 \mathrm{~m}$. Between $800-2400 \mathrm{~m}$, the number of anomalies occurring was steady and then a further reduction in the number of anomalies occurring was recorded at last $400 \mathrm{~m}$ of the pipeline. Figure 8 indicates that the immediate environment of the first $400 \mathrm{~m}$ of the pipeline encourages more corrosion while the last $400 \mathrm{~m}$ resist corrosion. The plot above indicates that the pipeline passes through three (3) separate environments. Figure 9 show the peak depth distribution along the pipeline. The plot shows that all peak depth (metal loss) recorded are all external, indicating no internal metal loss in the pipeline.

Figure 10 show the plot of the identified anomalies and their number of occurrences. It is seen that the only identified anomaly is "General Corrosion" which from Figure 9 occurred at the external surface of the pipeline. It shows 10 number of occurrences with the highest occurrence of 3at distance of $2800 \mathrm{~m}$. 


\section{Conclusion}

Pipeline intelligent pigging data were successfully collected from pigging data; operating from the Mimbo - TMMP pipeline MFL ultrasonic data. The minimum thickness, the average metal loss, the average metal loss before and after intelligent pigging, the percentage increase and average percentage increase, the estimated repair factor, the estimated life calculation, the metal loss peak depth distribution, surface location of the anomalies found were analyzed and compared with design specification and standards to determine the state of the pipelines and also the service condition of the pipeline. From the MFL data obtained, the average metal loss for the third pigging period was $1.1887 \mathrm{~mm}$, which is less than the corrosion allowance from design specification. There was a reduction in the percentage increase in average metal loss from $36.3 \%$ to $32.29 \%$. The MFL intelligent pigging reduced the metal loss in the pipeline operating three (3) phase crude from Mimbo-TMMP by $17.11 \%$ between 2005 and 2015 pigging period. The "dig-up sheet" showed the "feature type", "anomaly dimension", "feature identification", "surface location" and "estimated repair factor (ERF)" of the pipeline. All external anomalies have an ERF value $>0.2$, while the internal anomalies have an internal value of $\leq 0.2$. From the analysis of the MFL data obtained, the pipeline is deemed fit for continued service operation. The analysis of ultrasonic pig data obtained from the in-line inspection of the pipeline transporting gas from Mimbo - TMMP showed that the corrosion rate and estimated remaining life of the pipeline was 86years and 4 months. The "dig-up sheet" data showed no internal anomaly and the maximum defect found was $14.6 \%$ wall loss or remaining thickness of $6.7 \mathrm{~mm}$. The "dig-up sheet" showed the "anomaly dimension class", "peak depth", "surface location" and "estimated repair factor (ERF)" of the pipeline. All anomalies were externally located and have an ERF value of $\leq 0.2$. The analysis of the pigging data of the $4 \mathrm{nch}$ Mimbo - TMMP gas pipeline reveal that the pipeline is safe for continued service operation.

\section{References}

[1] Pharris T.C and Kolpa R.L (2007). "Overview of the Design, Construction, and Operation of Interstate Liquid Petroleum Pipelines”. ANL/EVS/TM/08-1.

[2] Obinna Aronu Kingsley (2017). "Integrity Management in the Energy Sector: An investigation of oil and gas assets". Norwegian University of Science and Technology.

[3] Kenneth P.G. and Taylor J. (2015). "Safety in the Transportation of Oil and Gas: Pipelines or Rail?". Fraser Research Bulletin.

[4] American Petroleum Institute, (1991). Pipeline Maintenance Welding Practices, 3rd edition, API RP 1107, American Petroleum Institute, Washington, D.C.

[5] Kishawy H.A, Gabbar H.A, (2010). "Review of pipeline integrity management practices". International Journal Press Vol. 87. Pages: 373-380. DOI:10.1016

[6] Baker Jr., M. (2009). "Mechanical Damage Final Report". Rep. No. DTRS56-02-D-7003, U.S. Department of Transportation Pipeline and Hazardous Materials Safety Administration Office of Pipeline Safety, Washington, DC.

[7] Menno, T., Van, O.O., Mastrigt, P., Francis, A. (2006). “An External Corrosion Direct Assessment Module for A Pipeline Integrity Management System”, 6th International Pipeline Conference, September 25-29, Calgary, Alberta, Canada.

[8] Papavinasam, S., Doiron, A, Revie, R.W. (2006). "Integrity Management of New Pipelines: Internal Corrosion Control, CORROSIONr5xa/2006, paper no. 187. Houston, TX: NACE. 